\title{
ON A SPECIAL CLASS OF ABELIAN FUNCTIONS
}

\author{
O. F. G. SCHILLING
}

In the applications of the theory of Riemann matrices to algebraic geometry and arithmetic it is necessary to emphasize the rings of complex multiplications in preference to the rational algebra associated to a given Riemann matrix. In recent years A. A. Albert completed and amplified the algebraic theory of Riemann matrices that was inaugurated by G. Scorza, S. Lefschetz, and others. ${ }^{1}$ We shall construct in this note the fields of abelian functions of genus $n$ whose multiplication rings contain a given order of a totally real field of absolute degree $n$. For the proof we shall compare two types of Riemann matrices defined by S. Lefschetz and O. Blumenthal. ${ }^{2}$ Applying equivalences to these Riemann matrices we find suitable matrices which show explicitly how a permissible ring of complex multiplications can be realized.

Let $k=R(\alpha)$ be a totally real field of degree $n$. Suppose that $\alpha=\alpha^{(1)}, \alpha^{(2)}, \cdots, \alpha^{(n)}$ are the $n$ conjugate roots of the defining irreducible equation $f(\alpha)=0$. Lefschet $z^{3}$ has determined the form of the most general Riemann matrix $\Omega_{1}$ of genus $n$ whose multiplication algebra $\mathfrak{\Re}\left(\Omega_{1}\right)$ contains an isomorphic image of the field $k$. The matrix $\Omega_{1}$ is equal to

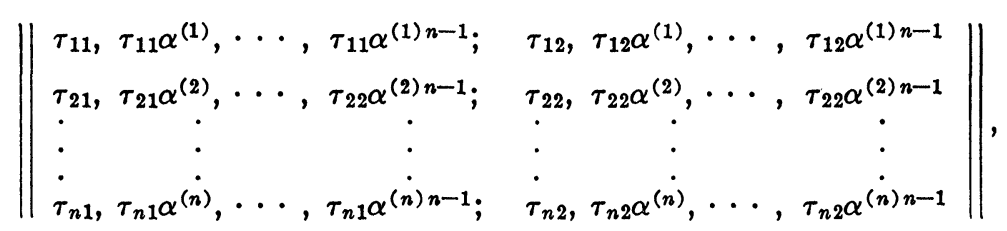

where $\tau_{11}, \cdots, \tau_{n 1}, \tau_{12}, \cdots, \tau_{n 2}$ are complex parameters subject to the restriction that $\Omega_{1}$ satisfy the defining equations of a Riemann matrix.

Now suppose that $\alpha_{1}=\alpha_{1}^{(1)}, \cdots, \alpha_{n}=\alpha_{n}^{(1)}$ is a basis of $k / R$. Let $A_{i}^{(j)}$ be the minors of the determinant $\delta=\delta^{(1)}=\left|\alpha_{i}^{(j)}\right|$. Then there ex-

Received by the editors July 26, 1944.

${ }^{1}$ For references see Solomon Lefschetz, Selected topics on algebraic geometry, National Research Council Bulletin vol. 63 (1928) pp. 310-395, and A. A. Albert, Structure of Algebras, Amer. Math. Soc. Colloquium Publications, vol. 24, 1939.

2 Solomon Lefschetz, On certain numerical invariants of algebraic varieties with applications to abelian varieties, Trans. Amer. Math. Soc. vol. 22 (1921) pp. 327-482 (quoted as L); Otto Blumenthal, Über Thetafunktionen und Modulfunktionen mehrerer Veräderlicher, Jber. Deutschen Math. Verein. vol. 13 (1904) pp. 120-132 (quoted as $\mathrm{B})$.

${ }^{3}$ L, loc. cit. p. 397. 
ist infinitely many numbers $\zeta=\zeta^{(1)}$ of $k$ so that $\zeta^{(1)} A_{1}^{(1)} / \delta^{(1)}$ $=\beta_{1}^{(1)}, \cdots, \zeta^{(1)} A_{n}^{(1)} / \delta^{(1)}=\beta_{n}^{(1)}$ form the basis of an ideal $\mathfrak{b}=\mathfrak{b}^{(1)}$ of $k .{ }^{4}$ The Riemann matrix defined by Blumenthal ${ }^{5}$ can then be written in the form

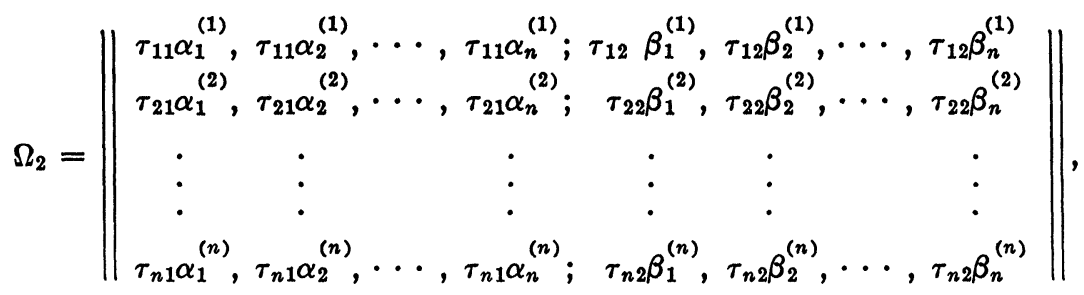

where $\tau_{j 2} / \tau_{j 1}, j=1, \cdots, n$, are restricted by the inequalities $R\left[\left(\tau_{j 2} / \tau_{j 1}\right) / \zeta^{(j)}\right]>0$.

\section{LEMMA 1. The matrices $\Omega_{1}$ and $\Omega_{2}$ are isomorphic.}

Proof. We first observe that $R\left(1, \alpha^{(1)}, \cdots, \alpha^{(1) n-1}\right)=R\left(\alpha_{1}^{(1)}, \cdots\right.$, $\left.\alpha_{n}^{(1)}\right)=R\left(\beta_{1}^{(1)}, \cdots, \beta_{n}^{(1)}\right)$. Hence there exist two regular $n \times n$ mattrices $\left\|a_{i j}\right\|$ and $\left\|b_{i j}\right\|$ in $R$ for which $\left(1, \alpha^{(1)}, \cdots, \alpha^{(1) n-1}\right)\left\|a_{i j}\right\|$ $=\left(\alpha_{1}^{(1)}, \alpha_{2}^{(1)}, \cdots, \alpha_{n}^{(1)}\right)$ and $\left(1, \alpha^{(1)}, \cdots, \alpha^{(1) n-1}\right)\left\|b_{i j}\right\|=\left(\beta_{1}^{(1)}, \beta_{2}^{(1)}\right.$, $\left.\cdots, \beta_{n}^{(1)}\right)$, respectively. Consequently

$$
\Omega_{1}\left\|\begin{array}{cc}
\left\|a_{i j}\right\| & 0 \\
0 & \left\|b_{i j}\right\|
\end{array}\right\|=\Omega_{2},
$$

in other words, $\Omega_{1}$ and $\Omega_{2}$ are isomorphic Riemann matrices. ${ }^{6}$

We next describe the explicit realization of $k$ as a field of multiplications of the matrix $\Omega_{2}$. Let $\gamma^{(1)}=\gamma$ be an element of $k$ and set $\left(\alpha_{1}^{(j)}, \alpha_{2}^{(j)}, \cdots, \alpha_{n}^{(j)}\right)=\mathfrak{a}^{(j)},\left(\beta_{1}^{(j)}, \beta_{2}^{(j)}, \cdots, \beta_{n}^{(j)}\right)=\mathfrak{b}^{(j)}, j=1, \cdots, n$. Then $\gamma^{(j)} \mathfrak{a}^{(j)}=\mathfrak{a}^{(j)} C(\mathfrak{a}, \gamma)$ and $\gamma^{(j)} \mathfrak{b}^{(j)}=\mathfrak{b}^{(j)} C(\mathfrak{b}, \gamma)$ where $C(\mathfrak{a}, \gamma)$ and $C(\mathfrak{b}, \gamma)$ are regular $n \times n$ matrices of $R$. Consequently

$$
\operatorname{diag}\left(\gamma^{(1)}, \gamma^{(2)}, \cdots, \gamma^{(n)}\right) \Omega_{2}=\Omega_{2}\left\|\begin{array}{cc}
C(\mathfrak{a}, \gamma) & 0 \\
0 & C(\mathfrak{b}, \gamma)
\end{array}\right\|^{7}
$$

The full algebra of multiplications $\mathfrak{A}\left(\Omega_{2}\right)$ is simple. In case $\mathfrak{A}\left(\Omega_{2}\right)$ contains elements satisfying an irreducible equation of degree $2 n \mathrm{it}^{\mathrm{t}}$ must be a totally imaginary extension $K=k\left(\mu^{1 / 2}\right), \mu \ll 0$ in $k .^{8}$

${ }^{4}$ Georg Landsberg, Ueber das Analogon des Riemann-Rach'schen Satzes in der Theorie der Algebraischen Zahlen, Math. Ann. vol. 50 (1897-1898) pp. 577-582.

5 B, loc. cit. p. 122.

${ }^{6} \mathrm{~L}$, loc. cit. p. 365.

${ }^{7}$ We use diag $\left(\gamma^{(1)}, \cdots, \gamma^{(n)}\right)$ for the $n \times n$ matrix whose diagonal elements are $\gamma^{(1)}, \cdots, \gamma^{(n)}$.

${ }^{8} \Delta \ll 0$ means that $\Delta$ and all its conjugates are negative. 
By the general theory of Riemann matrices the algebra $\mathfrak{A}\left(\Omega_{2}\right)$ has a representation by $2 n \times 2 n$ matrices in $R .{ }^{9}$ Hence each element of $\mathfrak{A}\left(\Omega_{2}\right)$ is the root of an equation of degree $2 n$ over $R$. Since $\mathfrak{A}\left(\Omega_{2}\right) \supseteq k$ there are multiplications satisfying an irreducible equation of degree $n$. The algebra $\mathfrak{A}\left(\Omega_{2}\right)$ certainly cannot contain a real multiplication satisfying an irreducible equation of degree $2 n$ for the roots of the characteristic equation must be double in case they are real. ${ }^{10}$ Since $\Omega_{2}$ admits a "general projectivity" $\alpha \in k$ the general characteristic equation cannot be the product of relative prime factors. ${ }^{11} \mathrm{Hence}$ the algebra $\mathfrak{A}\left(\Omega_{2}\right)$ cannot be semi-simple, that is, the direct sum of at least two simple rational algebras. Consequently $\Omega_{2}$ must be isomorphic to a matrix diag $(\omega, \cdots, \omega)$. The number of diagonal elements can at most be equal to two for otherwise the field $k$ would have a rational representation of degree less than $n$. In case that

$$
\Omega_{2} \cong\left\|\begin{array}{cc}
\omega & 0 \\
0 & \omega
\end{array}\right\|
$$

the algebra $\mathfrak{A}\left(\Omega_{2}\right)$ would be the algebra of square matrices over $k$. Then $\mathfrak{A}\left(\Omega_{2}\right)$ would contain all quadratic extensions over $k$, in particular a totally real extension of degree $2 n$ over $R$. This is impossible as was observed at the beginning of the proof. Thus there remains only $\mathfrak{A}\left(\Omega_{2}\right) \cong k\left(\mu^{1 / 2}\right), \mu \ll 0$.

In order to realize $k$ as the multiplication algebra of a Riemann matrix (real case) we let $\zeta$ be equal to the absolute value of the discriminant $^{12} \delta^{2}$, select $\tau_{j 1}=1, j=1, \cdots, n$, and take for $\tau_{j 2}$, $j=1, \cdots, n$, a set of transcendental numbers with positive imaginary parts. In case $\mathfrak{A}\left(\Omega_{2}\right)=K$ (imaginary case) let $\tau_{j 1}=1, j=1, \cdots, n$, and $\tau_{j 2}={ }_{+}\left(\mu^{(j)}\right)^{1 / 2}=\mathbf{M}^{(j)}, \mu \ll 0$ in $k$. Then $\Omega_{2}=\Omega_{2}(\mu)$ has the form

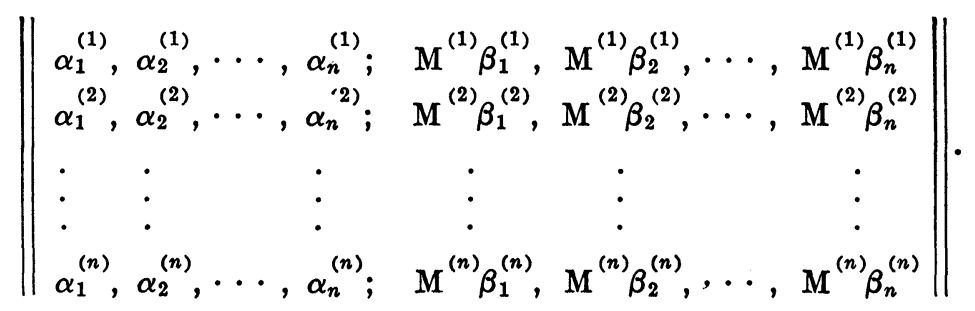

Observe now that the first row of $\Omega_{2}(\mu)$ represents a basis of the field $K=k(\mathbf{M})$. Hence $\mathbf{M}\left(\alpha_{1}^{(1)}, \ldots, \alpha_{n}^{(1)} ; \mathbf{M}^{(1)} \beta_{1}^{(1)}, \ldots, \mathbf{M}^{(1)} \beta_{n}^{(1)}\right)$

\footnotetext{
${ }^{9} \mathrm{~L}$, loc. cit. p. 392.

${ }^{10} \mathrm{~L}$, loc. cit. p. 392.

${ }^{11} \mathrm{~L}$, loc. cit. pp. 395, 397.

${ }^{12} \mathrm{~B}$, loc. cit. p. 122.
} 
$\left(\alpha_{1}^{(1)}, \ldots, \alpha_{n}^{(1)} ; \mathbf{M}^{(1)} \beta_{1}^{(1)}, \ldots, \mathbf{M}^{(1)} \beta_{n}^{(1)}\right)\left\|m_{i j}\right\|$ where the matrix $\left\|m_{i j}\right\|$ is a regular representation of $\mathbf{M}$ in $R$. Consequently $\operatorname{diag}\left(\mathbf{M}^{(1)}, \ldots, \mathbf{M}^{(n)}\right) \Omega_{2}(\mu)=\Omega_{2}(\mu)\left\|m_{i j}\right\|$, that is, $\Omega_{2}(\mu)$ admits $K=k(\mathbf{M})$ for its multiplication algebra.

THEOREM. In the real case there exists for each order of of maximal rank in $k$ at least one Riemann matrix which admits for its ring of multiplications the order $\mathbf{D}$. In the imaginary case there exists for each order $\mathfrak{O}$ of maximal rank in $K=k(\mathrm{M})$ at least one Riemann matrix which admits for its ring of multiplications the order $\mathfrak{D}$.

Proof. Let $\Omega_{2}$ be a Riemann matrix which has $\mathfrak{A}\left(\Omega_{2}\right) \cong k$. Suppose that $\omega_{1}, \cdots, \omega_{n}$ is a basis of $\mathrm{o}$ over the ring of integers. Then there exist rational regular $n \times n$ matrices $S_{1}$ and $S_{2}$ so that $\left(\omega_{1}, \cdots, \omega_{2}\right) S_{1}=\mathfrak{a}$ and $\left(\omega_{1}, \cdots, \omega_{2}\right) S_{2}=\mathfrak{b}$. Consider the matrix

$$
\Omega_{2}\left\|\begin{array}{ll}
S_{1} & 0 \\
0 & S_{2}
\end{array}\right\|
$$

it is isomorphic to $\Omega_{2}$ and admits exactly $\mathfrak{D}$ for its ring of multiplications. For this observe that $\phi\left(\omega_{1}, \cdots, \omega_{n}\right)=\left(\omega_{1}, \cdots, \omega_{n}\right)\left\|f_{i j}\right\|$ with $\phi \in k, \phi \notin 0$ gives rise to a matrix $\left\|f_{i j}\right\|$ whose elements do not all lie in the ring of ordinary integers since $\left(\omega_{1}, \cdots, \omega_{n}\right)$ is a basis of $\mathfrak{O}$.

Now let $\Omega_{2}(\mu)$ be a Riemann matrix admitting $K=k(\mathbf{M})$ for its algebra of multiplications. We suppose that $\Omega_{2}(\mu)$ is given as in the proof of Lemma 2. Then the first row of $\Omega_{2}(\mu)$ is a basis of $K$ over $R$. Let $\left(\omega_{1}, \cdots, \omega_{2 n}\right)$ be a basis of the given order $\mathfrak{D}$ in $K$. Then there exists a regular rational matrix $S$ so that $\Omega_{2}(\mu) S$ has $\left(\omega_{1}, \cdots, \omega_{2 n}\right)$ for its first row. Again $\Omega_{2}(\mu) S$ is isomorphic to $\Omega_{2}(\mu)$ and admits exactly $\mathfrak{D}$ for its ring of multiplications, that is, $\Phi \Omega_{2}(\mu) S=\Omega_{2}(\mu) S\left\|F_{i j}\right\|$ gives rise to a non-integral matrix $\left\|F_{i j}\right\|$ in case $\Phi \in K, \Phi \notin \mathcal{D}$.

COROLLARY. In both the real and imaginary case there exist fields of abelian functions $A$ of genus $n$ which have models whose rings of multiplications can be prescribed.

For the proof we refer to the construction of the fields $A$ for a given matrix of periods. ${ }^{13}$

The University of Chicago 370.

${ }^{13}$ A. Krazer, Lehrbuch der Thetafunktionen, Leipzig, 1903 and L, loc. cit. pp. 367- 